\title{
Binary Cyclic Pearson Codes
}

\author{
Ari Dwi Hartanto ${ }^{1^{*}}$, Al. Sutjijana ${ }^{2}$ \\ ${ }^{1,2}$ Department of Mathematics, Universitas Gadjah Mada, Yogyakarta, Indonesia
}

Article history:

Received Sep 2, 2019

Revised Feb 4, 2021

Accepted Mar 3, 2021

\section{Kata Kunci:}

Jarak Pearson, Kode

Pearson, Kode siklis

\section{Keywords:}

Pearson distance, Pearson code, Cyclic code

\begin{abstract}
Abstrak. Fenomena gain atau offset yang tidak terduga pada sistem komunikasi dan media penyimpan data modern seperti media penyimpanan berjenis optik (CD) dan memori non-volatile (flash) merupakan gangguan yang serius. Permasalahan ini dapat ditangani dengan mengaplikasikan jarak Pearson pada detektor error pada sistem tersebut karena jarak Pearson menawarkan kekebalan terhadap gain dan offset yang tidak menentu. Jarak ini hanya dapat digunakan pada suatu himpunan codewords tertentu, yaitu himpunan Pearson/kode Pearson. Salah satu contoh kode Pearson dapat ditemukan di kelas kode $T$-constrained. Dalam paper ini, diberikan kode 2-constrained biner dengan sifat siklis. Konstruksi kode ini diadopsi dari konstruksi pada kode siklis, akan tetapi kode yang dihasilkan tidak dapat dipandang sebagai kode siklik.
\end{abstract}

\begin{abstract}
The phenomena of unknown gain or offset on communication systems and modern storage such as optical data storage and non-volatile memory (flash) becomes a serious problem. This problem can be handled by Pearson distance applied to the detector because it offers immunity to gain and offset mismatch. This distance can only be used for a specific set of codewords, called Pearson codes. An interesting example of Pearson code can be found in the $T$-constrained code class. In this paper, we present binary 2constrained codes with the cyclic property. The construction of this code is adopted from cyclic codes, but it cannot be considered as cyclic codes
\end{abstract}

How to cite:

A. D. Hartanto and Al. Sutjijana, "Binary Cyclic Pearson Codes", J. Mat. Mantik, vol. 7, no. 1, pp. 1-8, May 2021.

\section{CONTACT:}

Ari Dwi Hartanto, $\boldsymbol{\nabla}$ ari@ugm.ac.id $\$$ Department of Mathematics, Universitas Gadjah Mada, Sleman, 


\section{Introduction}

Based on [1] and [2], gain and/or offset mismatch often occur on modern storages and communication channels. In non-volatile memories, for instance: a flash memory, the data is stored in a floating gate. It can leak away from the floating gate and affects a shift of the offset. In optical disc media, the signal retrieved by the sensor depends on the dimensions of the written features and the quality of the light path. The quality of the light path usually occurs because of fingerprints and scratches on the disc surface. These lead to gain and offset mismatch of the retrieved signal. Regarding this problem, Weber et.al. [3] presented a system code which involves the Person distance.

Pearson distance is resistant to gain and offset mismatch, so it can be an alternative to the Euclidean distance for improving the error performance of noisy channels with unknown gain and offset. Unfortunately, Pearson distance cannot be used for arbitrary sets of codewords, but it can only be used for a Pearson set/Pearson code, a set of codewords with special properties. Weber, Swart, and Immink [3] stated that one of the known Pearson code is $T(n, q)$. This code is a member of the $T$-constrained code class which consists of sequences in which each of $T$ pre-determined reference symbols appears at least once ([1], [3], [4]).

Let $C \subseteq Q^{n}$ be a $q$-ary code of length $\mathrm{n}$, where $Q=\{0,1, \cdots, q-1\}$ is the code alphabet of size $q \geq 2$. The alphabet symbols in codebook $C$ will not be treated as elements of $Z_{q}$, but as just integers. The reader should be careful that this situation will be different when we discuss linear codes in this paper.

Weber, Swart, and Immink ([3], [5]) presented two coding procedures, i.e. fixed-tofixed (FF) and variable-to-fixed (VF) length coding schemes which are special codes of $T(n, q)$. We present another special code of $T(n, q)$, that is Binary Cyclic Pearson Codes. We focus on how to construct the Pearson codes $C$ in which the codewords have cyclic property, that is, if $\left(a_{0}, a_{1}, \cdots, a_{n-1}\right) \in C$ then $\left(a_{n-1}, a_{0}, a_{1}, \cdots, a_{n-2}\right) \in C$. Our idea is taking the similar way of cyclic code construction (see [6] and [7]) for constructing a cyclic Pearson code. Cyclic code is one of the most important classes of linear codes. The cyclic codes have a rich algebraic structure and can be efficiently implemented using simple shift registers [8]. Although the cyclic codes possess algebraic structure, we do not emphasize algebraic structure on the binary cyclic Pearson codes. We only concern on the set of codewords that have cyclic property and satisfy the axioms of Pearson code. We investigate whether it can be constructed and the simple encoding scheme with a generator matrix can be built in a similar way on the simple encoding scheme of cyclic codes.

The rest of this paper is organized as follows. We start our presentation with a brief introduction to Pearson Distance, Pearson codes, and T-constrained codes in Section 2. In Section 3, we give a brief exposition of the cyclic codes, and then present binary cyclic Pearson codes. Finally, we draw some conclusions in Section 4.

\section{Preliminaries}

We first introduce a necessary notation used in this paper. For simplicity, we use the shorthand notation $\alpha \boldsymbol{u}+\beta=\left(\alpha u_{1}+\beta, \alpha u_{2}+\beta, \cdots, \alpha u_{n}+\beta\right)$ as described in [3]. The bold letter indicates a vector that can mean either a message word or a codeword. Suppose a codeword $\boldsymbol{c}$ is sent, and then a vector $\boldsymbol{r}=a(\boldsymbol{c}+\boldsymbol{v})+b$ is received, where $a, b \in R, a>$ 0 , and $v=\left(v_{1}, \cdots, v_{n}\right), v_{i} \in R$. The real numbers $a$ and $b$ are called gain and offset, respectively, and the entries of vector $v$ are noise sample from a zero-mean Gaussian distribution. The gain and offset will not affect sent codewords if we use Pearson distance detection ([9], [10]).

The following is the Pearson distance that is a well-known concept in statistics. Let $\boldsymbol{x}$ be a vector in $R^{n}$. Define: 
and

$$
\overline{\boldsymbol{x}}=\frac{1}{n} \sum_{i=1}^{n} x_{i}
$$

$$
\sigma_{x}^{2}=\sum_{i=1}^{n}\left(x_{i}-\bar{x}\right)^{2}
$$

The Pearson correlation coefficient of $\boldsymbol{x}$ and $\boldsymbol{y}$ is defined by

$$
\rho_{x, y}=\frac{\sum_{i=1}^{n}\left(x_{i}-\overline{\boldsymbol{x}}\right)\left(y_{i}-\overline{\boldsymbol{y}}\right)}{\sigma_{x} \sigma_{y}} .
$$

Next, the Pearson distance is defined as follow:

$$
\delta(\boldsymbol{x}, \boldsymbol{y})=1-\rho_{x, y} .
$$

To know the bound of $\delta(\boldsymbol{x}, \boldsymbol{y})$, we first note that $-1 \leq \rho_{x, y} \leq 1$. This implies that $0 \leq$ $\delta(\boldsymbol{x}, \boldsymbol{y}) \leq 2$.

By nearest neighbour decoding, a received vector will be decoded to the codeword closest to it, with respect to Pearson distance. If we have a received vector $\boldsymbol{r}$, the minimum Pearson distance detector outputs the codeword:

where $S$ is the codebook.

$$
\boldsymbol{c}_{\mathbf{0}}=\arg \arg \min _{c \in S} \delta(\boldsymbol{r}, \boldsymbol{c}),
$$

We can observe that for all $\alpha, \beta \in R, \alpha>0$, we have $\rho_{x, \alpha y+\beta}=\rho_{x, y}$. The equation implies that $\delta(\boldsymbol{x}, \alpha \boldsymbol{y}+\beta)=\delta(\boldsymbol{x}, \boldsymbol{y})$. It means that the Pearson distance is invariant under translation and scale. As a result, the minimum Pearson distance detector is immune to gain and offset mismatch. However, this arises a weakness. We cannot use the minimum Pearson distance detector for arbitrary codebooks because it cannot distinguish between vector $\boldsymbol{x}$ and $\boldsymbol{y}=\alpha \boldsymbol{x}+\beta$ where $\alpha, \beta \in R, \alpha>0,(\alpha, \beta) \neq(1,0)$, or mathematically, it can be written as

$$
\delta(\boldsymbol{r}, \boldsymbol{x})=\delta(\boldsymbol{r}, \boldsymbol{y})
$$

if $\boldsymbol{y}=\alpha \boldsymbol{x}+\beta$, for all $\alpha, \beta \in R$ with $\alpha>0$ and $(\alpha, \beta) \neq(1,0)$. Because of this fact, our codebook $C$ should has property that if $c \in C$ then $\alpha c+\beta \notin C$ for all $\alpha, \beta \in R$ with $\alpha>0$ and $(\alpha, \beta) \neq(1,0)$. Moreover, another property that has to be satisfied by codebook $C$ is that vectors in the form of $\boldsymbol{x}=(a, a, \cdots, a)$ is not allowed in the codebook $C$ since the vectors will lead to an undefined Pearson correlation coefficient.

Definition 1. Let $C$ be a nonempty subset of $Q^{n}$. The set $C$ is called Pearson code (or Pearson set) if it satisfies the following properties:

a. If $c \in C$ then $\alpha c+\beta \notin C$ for all $\alpha, \beta \in R$ with $\alpha>0$ and $(\alpha, \beta) \neq(1,0)$.

b. $\boldsymbol{x}=(a, a, \cdots, a) \notin C$ for all $a \in R$.

To have an example of Pearson codes, we first give a description of $T$-constrained codes. Let $T$ be an integer with $1 \leq T<q$ and reference symbols $a_{1}, \cdots, a_{T}$ be element of $Q$. A $T$-constrained code [1], denoted by $S_{q, n}\left(a_{1}, \cdots, a_{T}\right)$, consist of all $q$-ary codewords of length $n,\left(x_{1}, \cdots, x_{n}\right)$, such that

$$
\#\left\{i: x_{i}=j\right\}>0 \text { for each } j \in\left\{a_{1}, \cdots, a_{T}\right\} .
$$

Note that not all $T$-constrained codes are Pearson codes. For $T=2$, we can see that both 2constrained code $S_{q, n}(0,1)$ and $S_{q, n}(0, q-1)$ are Pearson codes, but for $q \geq 5, S_{q, n}(0,2)$ is not a Pearson code since it does not meet the first property of Pearson code.

We now focus to discuss the specific case, that is the $T$-constrained code $S_{q, n}(0,1)$. This code is Pearson codes, and will be denoted by $T(n, q)$ as in [3]. Weber, Swart, and Immink [3] proposed easy coding procedures, i.e., fixed-to-fixed (FF) and variable-to-fixed (VF) length coding schemes, denoted by $T_{F F}(n, q)$ and $T_{V F}(n, q)$, respectively. The simple 
FF scheme is to fill the first $n-2$ positions in the codeword $c$ with information symbols and to fill the last two symbols for reference purpose, i.e., $c_{n-1}=0$ and $c_{n}=1$. For VF schemes, it can be described as follows.

a. Take $n-2$ information from the $q$-ary source, and then set these as $\left(c_{1}, c_{2}, \cdots, c_{n-2}\right)$.

b. If there exists $i, 1 \leq i \leq n-2$, such that $c_{i}=0$, then choose $c_{n-1}$ to be a new information symbol, otherwise set $c_{n-1}=0$.

c. If there exists $i, 1 \leq i \leq n-1$, such that $c_{i}=1$, then choose $c_{n}$ to be a new information symbol, otherwise set $c_{n}=1$.

The resulted codewords from both schemes are in $T(n, q)$ since it contains at least one symbol 0 and at least one symbol 1 .

\section{Cyclic Pearson Codes}

In this section, we intend to construct cyclic Pearson codes by using similar ways as construction of cyclic codes. Let $\mathrm{F}$ be a finite field of order q, and $V_{n}(F)=\left\{\left(x_{1} x_{2} \cdots x_{n}\right) \mid\right.$ $\left.x_{i}, \cdots, x_{n} \in F\right\}$ be a vector space over $\mathrm{F}$. A nonempty subset $\mathrm{C}$ of $V_{n}(F)$ is called cyclic code if it is a subspace and every $\left(a_{1} a_{2} \cdots a_{n-1} a_{n}\right) \in C$ implies $\left(a_{n} a_{1} a_{2} \cdots a_{n-1}\right) \in C$. Cyclic codes can be constructed by ring polynomial approach.

Clearly, $V_{n}(F)$ is an abelian group under vector addition, but it is not a ring since we have not had a multiplication between any two vectors yet. In order to have multiplication operation such that $V_{n}(F)$ is a ring, the easiest way is to associate vectors in $V_{n}(F)$ with polynomials in $F[x]$, that is, if $\boldsymbol{a}=\left(a_{0} a_{1} \cdots a_{n-1}\right) \in V_{n}(F)$, then let

$$
a(x)=a_{0}+a_{1}+\cdots+a_{n-1} x^{n-1} .
$$

We select the polynomial $f(x)=x^{n}-1 \in F[x]$ to have a quotient ring $F[x] /\langle f(x)\rangle$. For all $\boldsymbol{a}, \boldsymbol{b} \in V_{n}(F)$, let $a(x) b(x)=v(x)$ where $v(x)$ is the polynomial of least degree in the equivalence class $[a(x) b(x)]$ of $F[x] /\langle f(x)\rangle$. Note that $v(x)$ is the remainder polynomial when $a(x) b(x)$ is divided by $f(x)$, and it is a polynomial of degree at most $n-1$ over $\mathrm{F}$, which is associated with an element of $V_{n}(F)$. In conclusion, now we have multiplication between any two vectors in $V_{n}(F)$ which is defined by this way, and with the association between the vectors and polynomials, we can essentially think of $V_{n}(F)$ and $F[x] /\langle f(x)\rangle$ interchangeable. Moreover, we can prove that $V_{n}(F)$ is a ring.

To have cyclic property of vectors, the choice of the polynomial $f(x)=x^{n}-1$ is most suitable. Multiplication a polynomial $v(x)$ in $F[x] /\langle f(x)\rangle$ by x corresponds to a cyclic shift of $\mathrm{v}$. This results in a fundamental theorem in cyclic codes presented as follows. A nonempty subset $C$ of $V_{n}(F)$ is a cyclic code if only if the set of polynomials $I$ associated with $C$ is an ideal in the ring $F[x] /\left\langle x^{n}-1\right\rangle$. This property helps us in constructing cyclic codes. In fact, there is a unique monic polynomial of least degree that generates a nonzero ideal $I$ of $F[x] /\left\langle x^{n}-1\right\rangle$, i.e., a monic polynomial divisor of $f(x)=x^{n}-1$. Thus, there is a $1-1$ correspondence between cyclic codes in $V_{n}(F)$ and monic polynomials $g(x) \in$ $F[x]$ that divide $f(x)$.

A monic polynomial $g(x)$ divisor of $f(x)=x^{n}-1$ over $F$ having degree $n-k$ will become the generator for a cyclic code $C$ of dimension $k$ in $V_{n}(F)$. The encoder encodes the message polynomial $a(x)$ (of degree less than or equal to $k-1$ ) to the codeword $a(x) g(x) \in F[x] /\langle f(x)\rangle$. Thus, the generator matrix for cyclic code $C$ is given in terms of $g(x)$ by

$$
G=\left[\begin{array}{c}
g(x) \\
x g(x) \\
\vdots \\
x^{k-1} g(x)
\end{array}\right] .
$$

Note that $G$ is a matrix of $k \times n$ where the $i^{\text {th }}$ row of $G, 2 \leq i \leq k$, is the $i-1$ right shifts of the first row of $G$. 
We now turn our discussion to Pearson codes. For simplicity, sometimes we will write a vector in $V_{n}(F)$ or in codes $C$ in the form $a_{0} a_{1} \cdots a_{n}$ instead of $\left(a_{0}, a_{1}, \cdots, a_{n}\right)$. We note that the cyclic codes $C$ are not Pearson codes since $00 \cdots 0$ belongs to $C$. We observe some nonempty subsets of cyclic code $C$ such that it is Pearson codes. In case our discussion is in Pearson code, we treat the information symbols of codewords as real numbers instead of elements of a finite field.

In order to find a nonempty subset of cyclic codes $C$ such that it is a Pearson code, clearly, we should eliminate the zero vector from $C$ since it breaks the second axiom of the definition of Pearson codes. We should observe whether there exist other elements having form $a a \cdots a$ in $C$. Let us see a $(7,4)$-cyclic code over $Z_{2}$ generated by $g(x)=1+x+x^{3}$. The generator matrix of code $C$ is

$$
\begin{aligned}
& G=\left[1+x+x^{3} x+x^{2}+x^{4} x^{2}+x^{3}+x^{5} x^{3}+x^{4}+x^{6}\right]
\end{aligned}
$$

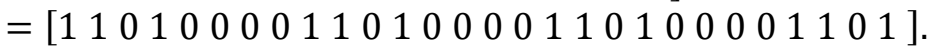

The message $\boldsymbol{m}=1011$ is encoded to be codeword $\boldsymbol{c}=\boldsymbol{m} G=1111111 \in C$. Such codeword must be omitted from $C$. It means that if our observation is in $(n, k)$-cyclic codes, we have to make attention for messages from which codewords having form $a a \cdots a$. We note that such messages are the polynomials which are quotient of division $h(x)=r+$ $r x+\cdots+r x^{n-1}$, where $r \in F$, by the generator polynomial $g(x)$ in $F[x] /\left\langle x^{n}-1\right\rangle$.

Clearly, if $c$ is a codeword of $(n, k)$-cyclic code $C$ over finite field $F$, then $\alpha c+$ $\left(1_{F}, 1_{F}, \cdots, 1_{F}\right)$ must be element of $C$, for all $\alpha \in N$. This does not meet the first axiom of the definition of Pearson codes. Now we go on a specific case, that is $(n, k)$-cyclic code $C$ over finite field $Z_{2}$. In this case, we have a good result. We note that $\left.C-\{00 \cdots 0,11 \cdots 1)\right\}$ is a 2-constrained code $S_{2, n}(0,1)$ if we treat the information symbols of codewords in $C$ as real numbers. In conclusion, we obtain that the code $P=C-\{00 \cdots 0,11 \cdots 1\}$ is a Pearson code, and we call this code the binary cyclic Pearson codes.

For a simple encoding scheme, we intend to use the generator matrix, but we face a problem when the message word is the zero vector. We should give a special treatment for this message word. First, we need to limit $n$ to an even positive integer. We correspond the zero vector with the codeword $1010 \cdots 10$. Since $1010 \cdots 10$ is a codeword, $0101 \cdots 01$ must be considered as a codeword. We correspond $0101 \cdots 01$ with a message word $a_{0} a_{1} \cdots a_{k-1}$ such that $a_{0} a_{1} \cdots a_{k-1} G=111 \cdots 1$. Unfortunately, we cannot guarantee that such a message word will exist. In order to guarantee the existence of such a message word, the polynomial $h(x)=1+x+\cdots+x^{n-1}$ must be divisible by the generator polynomial $g(x)$. By this condition, we have $a_{0}+a_{1} x+\cdots+a_{k-1} x^{k-1}$ is $h(x) / g(x)$. Next, we should be careful if $1010 \cdots 10$ belongs to $(n, k)$-cyclic code $C$. It should not be allowed since we have corresponded $1010 \cdots 10$ with message word $000 \cdots 0$.

Table 1. Some generator polynomials of binary cyclic Pearson codes

\begin{tabular}{lll}
\hline$n$ & $k$ & Generator Polynomial \\
\hline 2 & 1 & $g(x)=1+x$ \\
4 & 1 & $g(x)=(1+x)^{3}$ \\
6 & 5 & $g(x)=1+x$ \\
& 3 & $g(x)=(1+x)\left(1+x+x^{2}\right)$ \\
& 1 & $g(x)=(1+x)\left(1+x+x^{2}\right)^{2}$ \\
8 & 1 & $g(x)=(1+x)^{7}$ \\
10 & 9 & $g(x)=1+x$ \\
& 5 & $g(x)=(1+x)\left(1+x+x^{2}+x^{3}+x^{4}\right)$ \\
& 1 & $g(x)=(1+x)\left(1+x+x^{2}+x^{3}+x^{4}\right)^{2}$ \\
\hline
\end{tabular}


Table 1. Some generator polynomials of binary cyclic Pearson codes (continued)

\begin{tabular}{llc}
\hline$n$ & $k$ & Generator Polynomial \\
\hline 12 & 9 & $g(x)=(1+x)^{3}$ \\
& 7 & $g(x)=(1+x)^{3}\left(1+x+x^{2}\right)$ \\
5 & $g(x)=(1+x)^{3}\left(1+x+x^{2}\right)^{2}$ \\
3 & $g(x)=(1+x)^{3}\left(1+x+x^{2}\right)^{3}$ \\
& $g(x)=(1+x)^{3}\left(1+x+x^{2}\right)^{4}$ \\
\hline
\end{tabular}

Based on the explanation above, not all $(n, k)$-cyclic codes over $Z_{2}$ can be used for constructing a binary cyclic Pearson code. As summary, the following conditions must be held in order that $(n, k)$-cyclic codes over $Z_{2}$ can be taken for constructing a binary cyclic Pearson code:

a. $n$ is even.

b. The polynomial $h(x)=1+x+\cdots+x^{n-1}$ is divisible by the generator polynomial $g(x)$.

c. The polynomial $1+x^{2}+x^{4}+\cdots+x^{n-2}$ is not divisible by the generator polynomial $g(x)$.

By using MAGMA (Computational Algebra System) (see [11]), we observe all possibility of $(n, k)$-cyclic codes over $Z_{2}$, for $n=2,4, \cdots, 12$, that meets three conditions above. Table 1 gives all generator polynomials of binary cyclic Pearson codes for $n=2,4, \cdots, 12$.

Example 1. Consider $n=6, V_{6}\left(Z_{2}\right)$, and $f(x)=x^{6}-1$. We take polynomial $g(x)=1+$ $x$ as a generator of the binary cyclic Pearson code, so we have a generator matrix:

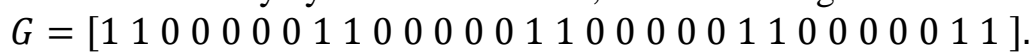

We divide $1+x+x^{2}+x^{3}+x^{4}+x^{5}$ by $g(x)$, and we have $1+x^{2}+x^{4}$ as a result. Then, the message words 00000 and 10101 are encoded to the codewords 101010 and 010101, respectively. For all $\boldsymbol{m} \in V_{5}\left(Z_{2}\right)-\{00000,10101\}, \boldsymbol{m}$ is encoded to codeword $\boldsymbol{m} G$. Therefore, the binary cyclic Pearson code of length $n=6$ and with generator $g(x)=$ $1+x$ is

where $P_{0}=\{101010,010101\}$.

$$
P=\left\{\boldsymbol{m} G \mid \boldsymbol{m} \in V_{5}\left(Z_{2}\right)-\{00000,10101\}\right\} \cup P_{0},
$$

\section{Conclusions}

A binary cyclic code of even length usually has a small Hamming distance. Some of the Hamming distances of cyclic codes can be seen on Table 2. A code with small distance can be said as a bad code because it can only detect and correct small errors. However, it does not mean that the code is always useless. As we explained above, an even length of code become one of the sufficient conditions for constructing a binary cyclic Pearson code. Therefore, some of cyclic codes of even length are needed for binary cyclic Pearson codes.

In this paper we have investigated binary cyclic Pearson codes for $n=2,4,6,8,10,12$, and the result is that the binary cyclic Pearson codes exist for each of these $n$. We have not yet investigated codes of even length generally. Our conjecture is the binary cyclic Pearson codes exist for all even positive integer $n$. 
Table 2. Some generators of $(n, k, d)$-cyclic codes and its compatibility for binary cyclic Pearson codes (BCPC)

\begin{tabular}{|c|c|c|c|c|}
\hline$n$ & $k$ & Generator Polynomial & $d$ & $\begin{array}{c}\text { Compatibility of generator } \\
\text { for BCPC }\end{array}$ \\
\hline 2 & 1 & $g(x)=1+x$ & 2 & yes \\
\hline \multirow[t]{3}{*}{4} & 3 & $g(x)=1+x$ & 2 & no \\
\hline & 2 & $g(x)=(1+x)^{2}$ & 2 & no \\
\hline & 1 & $g(x)=(1+x)^{3}$ & 4 & yes \\
\hline \multirow[t]{7}{*}{6} & 5 & $g(x)=1+x$ & 2 & yes \\
\hline & 4 & $g(x)=(1+x)^{2}$ & 2 & no \\
\hline & 4 & $g(x)=1+x+x^{2}$ & 2 & no \\
\hline & 2 & $g(x)=\left(1+x+x^{2}\right)^{2}$ & 3 & no \\
\hline & 3 & $g(x)=(1+x)\left(1+x+x^{2}\right)$ & 2 & yes \\
\hline & 2 & $g(x)=(1+x)^{2}\left(1+x+x^{2}\right)$ & 4 & no \\
\hline & 1 & $g(x)=(1+x)\left(1+x+x^{2}\right)^{2}$ & 6 & yes \\
\hline \multirow[t]{7}{*}{8} & 7 & $g(x)=(1+x)$ & 2 & no \\
\hline & 6 & $g(x)=(1+x)^{2}$ & 2 & no \\
\hline & 5 & $g(x)=(1+x)^{3}$ & 2 & no \\
\hline & 4 & $g(x)=(1+x)^{4}$ & 2 & no \\
\hline & 3 & $g(x)=(1+x)^{5}$ & 4 & no \\
\hline & 2 & $g(x)=(1+x)^{6}$ & 4 & no \\
\hline & 1 & $g(x)=(1+x)^{7}$ & 8 & yes \\
\hline \multirow[t]{7}{*}{10} & 9 & $g(x)=1+x$ & 2 & yes \\
\hline & 8 & $g(x)=(1+x)^{2}$ & 2 & no \\
\hline & 6 & $g(x)=1+x+x^{2}+x^{3}+x^{4}$ & 2 & no \\
\hline & 2 & $g(x)=\left(1+x+x^{2}+x^{3}+x^{4}\right)^{2}$ & 5 & no \\
\hline & 5 & $g(x)=(1+x)\left(1+x+x^{2}+x^{3}+x^{4}\right)$ & 2 & yes \\
\hline & 4 & $g(x)=(1+x)^{2}\left(1+x+x^{2}+x^{3}+x^{4}\right)$ & 4 & no \\
\hline & 1 & $g(x)=(1+x)\left(1+x+x^{2}+x^{3}+x^{4}\right)^{2}$ & 10 & yes \\
\hline \multirow[t]{14}{*}{12} & 11 & $g(x)=1+x$ & 2 & no \\
\hline & 10 & $g(x)=(1+x)^{2}$ & 2 & no \\
\hline & 9 & $g(x)=(1+x)^{3}$ & 2 & yes \\
\hline & 8 & $g(x)=(1+x)^{4}$ & 2 & no \\
\hline & 10 & $g(x)=1+x+x^{2}$ & 2 & no \\
\hline & 8 & $g(x)=\left(1+x+x^{2}\right)^{2}$ & 2 & no \\
\hline & 6 & $g(x)=\left(1+x+x^{2}\right)^{3}$ & 3 & no \\
\hline & 4 & $g(x)=\left(1+x+x^{2}\right)^{4}$ & 3 & no \\
\hline & 9 & $g(x)=(1+x)\left(1+x+x^{2}\right)$ & 2 & no \\
\hline & 7 & $g(x)=(1+x)\left(1+x+x^{2}\right)^{2}$ & 2 & no \\
\hline & 5 & $g(x)=(1+x)\left(1+x+x^{2}\right)^{3}$ & 4 & no \\
\hline & 3 & $g(x)=(1+x)\left(1+x+x^{2}\right)^{4}$ & 6 & no \\
\hline & 8 & $g(x)=(1+x)^{2}\left(1+x+x^{2}\right)$ & 2 & no \\
\hline & 6 & $g(x)=(1+x)^{2}\left(1+x+x^{2}\right)^{2}$ & 2 & no \\
\hline
\end{tabular}


Table 2. Some generators of $(n, k, d)$-cyclic codes and its compatibility for binary cyclic Pearson codes (BCPC) (continued)

\begin{tabular}{ccccc}
\hline$n$ & $k$ & Generator Polynomial & $d$ & $\begin{array}{c}\text { Compatibility of generator } \\
\text { for BCPC }\end{array}$ \\
\hline 12 & 4 & $g(x)=(1+x)^{2}\left(1+x+x^{2}\right)^{3}$ & 4 & no \\
& 2 & $g(x)=(1+x)^{2}\left(1+x+x^{2}\right)^{4}$ & 6 & no \\
& 7 & $g(x)=(1+x)^{3}\left(1+x+x^{2}\right)$ & 4 & yes \\
& 5 & $g(x)=(1+x)^{3}\left(1+x+x^{2}\right)^{2}$ & 4 & yes \\
3 & $g(x)=(1+x)^{3}\left(1+x+x^{2}\right)^{3}$ & 4 & yes \\
& 1 & $g(x)=(1+x)^{3}\left(1+x+x^{2}\right)^{4}$ & 12 & yes \\
\hline
\end{tabular}

\section{Acknowledgement}

We thanks to Jos Weber from Delft University of Technology (TU Delft) for the discussion and his papers shared with us.

\section{References}

[1] K. A. S. Immink and J. H. Weber, "Minimum pearson distance detection for multilevel channels with gain and/or offset mismatch," IEEE Trans. Inf. Theory, vol. 60, no. 10, pp. 5966-5974, 2014, doi: 10.1109/TIT.2014.2342744.

[2] F. Sala, K. A. S. Immink, and L. Dolecek, "Error Control Schemes for Modern Flash Memories," IEEE Consum. Electron., vol. 4, no. 1, pp. 66-73, 2015.

[3] J. H. Weber, K. A. S. Immink, and S. R. Blackburn, "Pearson codes," IEEE Trans. Inf. Theory, vol. 62, no. 1, pp. 131-135, 2016, doi: 10.1109/TIT.2015.2490219.

[4] K. A. S. Immink and J. H. Weber, "Hybrid Minimum Pearson and Euclidean Distance Detection," IEEE Trans. Commun., vol. 63, no. 9, pp. 3290-3298, 2015, doi: 10.1109/TCOMM.2015.2458319.

[5] J. H. Weber, T. G. Swart, and K. A. S. Immink, "Simple systematic Pearson coding," IEEE Int. Symp. Inf. Theory - Proc., vol. 2016-Augus, pp. 385-389, 2016, doi: 10.1109/ISIT.2016.7541326.

[6] S. A. Vanstone and P. C. Oorschot, An Introduction to Error Correcting Codes with Applications. Springer US, 1989.

[7] S. Ling and C. Xing, Coding Theory. Cambridge University Press, 2004.

[8] A. Betten, M. Braun, H. Fripertinger, A. Kerber, A. Kohnert, and A. Wassermann, Error-Correcting Linear Codes. Springer Berlin Heidelberg, 2006.

[9] K. A. S. Immink, "Coding schemes for multi-level channels with unknown gain and/or offset," IEEE Int. Symp. Inf. Theory - Proc., pp. 709-713, 2013, doi: 10.1109/ISIT.2013.6620318.

[10] K. A. S. Immink, "Coding schemes for multi-level Flash memories that are intrinsically resistant against unknown gain and/or offset using reference symbols," Electron. Lett., vol. 50, no. 1, pp. 20-22, 2014, doi: 10.1049/el.2013.3558.

[11] MAGMA Handbook, "http://magma.maths.usyd.edu.au/magma/handbook/ (accessed Nov. 05, 2018). 